Discussion Paper No. 914

HIERARCHIES VERSUS COMMITTEES:

COMMUNICATION

AND INFORMATION ACQUISITION

IN ORGANIZATIONS

Junichiro Ishida

September 2014

The Institute of Social and Economic Research

Osaka University

6-1 Mihogaoka, Ibaraki, Osaka 567-0047, Japan 


\title{
Hierarchies Versus Committees: Communication and Information Acquisition in Organizations*
}

\author{
Junichiro Ishida ${ }^{\dagger}$ \\ ISER, Osaka University
}

September 22, 2014

\begin{abstract}
In most firms, if not all, workers are divided asymmetrically in terms of authority and responsibility. In this paper, we view the asymmetric allocations of authority and responsibility as essential features of hierarchy and examine why hierarchies often prevail in organizations from that perspective. A key departure is that we consider a case where the authority relationship is defined only by the allocation of responsibility via contingent contracts. Within this framework, we show that the contractual arrangement which allocates responsibility asymmetrically often emerges as the optimal organizational form, which gives rise to the chain of command pertaining to hierarchical organizations.
\end{abstract}

JEL Classification Codes: D03, D99.

Key Words: Authority; Responsibility; Contract; Cheap-talk communication; Information acquisition.

${ }^{*}$ Correspondence to: Junichiro Ishida, Institute of Social and Economic Research, Osaka University, 6-1 Mihogaoka, Ibaraki, Osaka 567-0047, Japan. e-mail address: jishida@iser.osaka-u.ac.jp.

${ }^{\dagger}$ I thank an anonymous referee for many careful comments which substantially improve the exposition of the manuscript. All remaining errors are my own. The financial support from Grant-in-Aid for Scientific Research (C), JSPS, is gratefully acknowledged. 


\section{Introduction}

It is very rare, if ever, to find a firm where all of its workers are treated just equally. In most firm organizations, some workers, typically those higher up in the hierarchy, are conferred more authority and hence carry an asymmetrically large weight in the decision-making process than others. At the same time, those with more authority are also given more stringent incentives and moreover held responsible for a wider range of outcomes, such as the firm's or their respective division's overall performances. While the degree of centralization or worker empowerment differs across firms as well as over time, workers are by and large divided asymmetrically in terms of both authority and responsibility (i.e., accountability for outcomes). In this paper, we view these asymmetric allocations of authority and responsibility as essential features of hierarchical organizations and examine why hierarchies often prevail in organizations from that perspective.

At a glance, the asymmetric allocation of responsibility seems to be a straightforward consequence of that of authority because if a worker is entitled to make a decision, he should be held accountable for any consequences brought by that decision. In this line of reasoning, it is the allocation of authority which subsequently determines the allocation of responsibility, so that the causation runs from authority to responsibility. What is implicit in this argument is therefore that the principal can allocate (formal) authority at her own discretion. While this presumption may be reasonable in some contexts, one can also think of a case where the principal cannot explicitly contract on decision rights. In this paper, as a key departure from the existing literature, we consider an environment where the principal has no direct control over the allocation of authority, and the authority relationship is instead determined by the allocation of responsibility via contingent contracts. ${ }^{1}$ Since the presence of hierarchy is quite ubiquitous in firm organizations, it seems worthwhile to explore the nature of hierarchy in an environment where, due to some technological or informational constraints, the principal has no direct control over the allocation of authority.

To this end, we consider an organization which consists of a principal (the contract designer) and two agents. Each agent privately chooses a task to implement, which stochastically leads to some observable output. The productivity of each task is not known ex ante,

\footnotetext{
${ }^{1}$ In the literature, it is often assumed that the principal allocates (formal) authority at her own discretion, e.g., by granting or restricting access to critical resources that are indispensable for production (Aghion and Tirole, 1997; Rajan and Zingales, 2001; Dessein, 2002; Hart and Moore, 2005).
} 
and each agent must hence acquire information about which task is the more productive. This information-acquisition stage involves two rounds. In the first round, each agent privately exerts effort to acquire information about the productivity of each task (or produce an "idea"). This is followed by the second round where each agent sends a costless message to share this information, if any, with the other agent. With no feasible way to allocate authority, however, no party can force an agent to take actions that are not in his best interest, and every decision to be made must be incentive-compatible under the agreed contracts. The chain of command (i.e., who orders and who obeys) then arises endogenously as an optimal response to the given structure of incentives.

The current framework admits several organizational forms, depending on how the structure of incentives is shaped by the set of contingent contracts. We say that a hierarchy emerges when one agent (the superior) orders the other agent (the subordinate) what to do, and the subordinate has an incentive to follow the order. Under this contractual arrangement, the flow of information is restricted to be unilateral, always from the superior to the subordinate, and the superior is given a disproportionately large weight in the decision-making process ex ante. As an alternative to this arrangement, the incentive contracts may be designed to place no restriction over the flow of information. In this case, information flows from the informed party to the uninformed in an unspecified direction, and each agent is given an equal weight ex ante as in a committee. With the addition of the benchmark case, we consider and compare the following three organizational forms summarized as below. ${ }^{2}$

1. Independent Production (the benchmark): Each agent independently exerts effort to acquire the information without ever communicating with each other. No interactions take place between the agents.

2. Committee (the symmetric contracts): Both of the agents are induced to exert effort and then communicate with each other. Communication is bilateral where each agent carries the same weight ex ante in the decision-making process.

3. Hierarchy (the asymmetric contracts): Only one of the agents, the superior, is induced to exert effort while the other, the subordinate, is not. Communication is unilateral where the superior orders the subordinate what to do.

\footnotetext{
${ }^{2}$ While there are more feasible contractual arrangements in this setup, we can show that it suffices to consider those three cases. See Appendix B for more detail.
} 
We show that the optimal contractual arrangement is often asymmetric, where only one agent is motivated to acquire the information, and Hierarchy thus emerges as the optimal organization form under a wide range of circumstances. ${ }^{3}$ To derive this result, we focus on the tradeoff between costly information acquisition and costless (cheap-talk) communication, which stems from the fact that an incentive contract in this setup is subject to two constraints, one for information acquisition (whether to exert effort to acquire information) and the other for truthful communication (whether to reveal truthfully the acquired information). The problem is that these two constraints are naturally at odds with each other, because the constraint for information acquisition calls for competition between the agents while that for communication calls for cooperation between them. The optimal contract must balance these concerns, which in turn determines how the information is acquired and disseminated in the organization.

To illustrate the tradeoff, we start with the benchmark case of Independent Production which makes no use of communication. The optimal contract under this arrangement generically takes the form of relative performance evaluation, where the agents are compensated based on the difference in the outputs. This is the most efficient way to provide incentives if the principal's only concern is to motivate them to exert effort. Independent Production has a clear weakness, however, because the constraint for truthful communication cannot be satisfied under relative performance evaluation. With no information flowing between the agents, therefore, it may leave some of the existing knowledge unutilized: in this sense, the level of competition between the agents is often excessive under Independent Production, which impedes cooperation that is equally critical for efficient production. Given that information is free to disseminate once it is acquired, it is clearly ex post optimal to share any useful information between the agents. Since it is not known ex ante who actually ends up with a good idea, this can be done most effectively by removing any restrictions on the flow of information.

Given this result, we now turn to Committee where both of the agents are induced to exert effort and no restriction whatsoever is placed on the extent of communication (bilateral communication). Under this arrangement, each agent carries the same weight in the decision-

\footnotetext{
${ }^{3}$ The contrast between hierarchies and committees is also depicted in Sah and Stiglitz (1988) with a totally different approach. They compare three different decision-making protocols - committees, hierarchies and polyarchies - with its particular emphasis on the tradeoff between the type I and type II errors.
} 
making process ex ante, and information flows from a party with an idea to a party without it. A virtue of Committee is then evident: it can make the best use of the existing knowledge within the organization by fully exploiting the benefit of costless communication. As it turns out, though, this ex post optimal arrangement is not necessarily ex ante optimal, as it raises the agency cost of inducing costly effort. The analysis identifies three channels through which bilateral communication entails ex ante inefficiency, as summarized below.

Incentive provision: To facilitate communication, the agents must be held jointly accountable, which is a less efficient way to provide incentives.

Freeriding: When communication is informative, there arises an incentive to freeride on the other agent's effort.

Task coordination: When communication is restricted, the agents can increase the chance of coordinating their task choices only by acquiring the information. This effect is absent when the agents can always coordinate via communication.

Due to these problems, Committee is often less profitable, especially when the agency problem regarding information acquisition is sufficiently severe. This does not necessarily mean, though, that we must give up the benefit of communication entirely; it rather means that going all the way from no communication to bilateral communication is often excessive. We argue that there is a way to exploit the benefit of communication while keeping its cost minimum. This can be done by the asymmetric allocation of responsibility where one agent, the superior, is offered high-powered (team) incentives while the other, the subordinate, is offered low-powered (individual) incentives. This asymmetric contract yields two beneficial effects. First, Hierarchy reduces the total agency cost as it only needs to motivate one agent. In an environment where information acquisition is costly while communication is free, this is a thrifty way to acquire information as a group. Second, the asymmetric and concentrated allocation of responsibility eliminates the freeriding incentive as the superior can no longer rely on the subordinate's information. We show that this asymmetric contractual arrangement is optimal for a wide range of circumstances, as it achieves the right balance of competition and cooperation. Under the asymmetric allocation of responsibility, the flow of information is restricted to be unilateral, always from the superior to the subordinate, which endogenously gives rise to the chain of command that is inherent in hierarchical organizations.

The results obtained in this paper suggest that whether Hierarchy or Committee is optimal 
depends crucially on the severity of the agency problem in the information-acquisition stage. Hierarchy, which allocates responsibility asymmetrically, outperforms Committee when costly information acquisition is the main concern to be dealt with. We argue that this corresponds to typical firm organizations where a relatively fixed group of members must confront with and find solutions for new problems on the day-to-day basis. In such a case, Hierarchy is often optimal, lending support for the view that most firms are hierarchical. This stands in sharp contrast to committees which typically consist of experts who are well informed in the first place. In committees, members are in many cases selected for specific problems and information acquisition is hence rarely an issue. In such a case, there is no reason to treat members asymmetrically, and Committee with the more symmetric allocation of authority and responsibility emerges as the better option to cope with the problem at hand.

The paper is related to several stands of literature. ${ }^{4}$ The nature of authority we consider here is closely related to the notion of real authority by Aghion and Tirole (1997) in that the authority relationship stems from one's informational superiority over others. Aghion and Tirole (1997) consider an incomplete-contracting environment where the principal can only allocate decision rights and analyze how the allocation of formal authority affects the agents' incentive to acquire information through the tradeoff between the loss of initiative and the loss of incentive. ${ }^{5}$ There are two critical difference between their work and ours. First, we consider a multi-agent situation with emphasis on the authority relationship between the agents, rather than on that between the principal and the agent. Second, and more importantly, we consider an environment where the principal cannot even allocate formal authority: the structure of information is determined by the allocation of formal authority in Aghion and Tirole (1997), whereas it is determined by the allocation of responsibility via incentive contracts in the current analysis. ${ }^{6}$ We then focus on a different incentive problem

\footnotetext{
${ }^{4}$ Needless to say, there is a vast literature on hierarchy. A large body of literature centers around the Revelation Principle which implies that centralized contracting cannot be dominated by hierarchial contracting. In the face of this fact, a substantial amount of attention has been paid to the cases where the Revelation Principle fails to hold, e.g., due to costly communication/information processing or collusion among agents. Some of the most notable are Radner (1993), Van Zandt (1999), Baliga and Sjostrom (1998), Bolton and Dewatripont (1994), Laffont and Matimort (1998), Laffont et al. (2003). Mookherjee (2006) provides an excellent survey from this mechanism design perspective.

${ }^{5}$ See Choe and Ishiguro (2012) for a more recent example of this approach.

${ }^{6}$ In this respect, the paper is also related to the emerging literature which explores the nature of interpersonal authority. Van den Steen (2010) considers a setting where two agents engage in a pair of related projects, with decision externalities between the two projects. With differing priors, the agents can disagree about what decision to make. In this setup, he shows that how the allocation of assets and income rights influences the nature of interpersonal authority. In an environment where the agent can disobey the principal's order, Marino
} 
which stems from the tension between information acquisition and truthful communication.

Second, the current model builds on cheap-talk communication, initiated by Crawford and Sobel (1982), which deals with the transmission of unverifiable soft information. ${ }^{7}$ There are now many applications of this insightful idea, most notably Dessein (2002), and this paper belongs to this strand as well. ${ }^{8}$ In most applications, however, the payoff structure is exogenously given, despite the fact that the degree of preference incongruence is almost always the center of attention in cheap-talk models. Instead, we introduce incentive contracts which allow us to endogenize the degree of preference incongruence. By writing a contract, the principal can either facilitate or restrict communication between the agents. Of course, since the principal can only observe each agent's output and the incentive for information acquisition cannot hence be separated out from that for communication, any contract inevitably affects the process of information acquisition, leading to a tradeoff which lies at the core of the analysis.

Finally, the paper is also related to a growing literature on committees with endogenous information. As often emphasized in the literature (Li, 2001; Li and Suen, 2009), one of the important aspects of the model is that the ex post efficient rule is not necessarily ex ante efficient. The freeriding problem in a committee is pointed out by $\mathrm{Li}$ (2001) where each member must independently acquire information. ${ }^{9}$ In that environment, he shows that a super-majority rule, the one that biases against the ex ante preferred option, can be used to mitigate the freeriding incentive. The current model provides yet another example of this situation, illuminating the tradeoff between information acquisition and communication. A point of departure is that we introduce incentive contracts into the model and suggest a different solution to this problem. With the explicit consideration of incentive contracts, we also show that information acquisition in a committee entails different types of inefficiency, i.e., incentive provision and freeriding. ${ }^{10}$

et al. (2010) argue how the agent's ability to disobey the principal limits the extent of centralization. Neither work considers the moral hazard problem in information acquisition, however, as all the necessary information for the agents is exogenously given. See Bolton and Dewatripont (2013) for a succinct survey on this issue.

${ }^{7}$ There is also a strand of literature which deals with the disclosure of verifiable hard information. See Grossman and Hart (1980), Milgrom (1981) and Caillaud and Tirole (2007) for this approach.

${ }^{8}$ Also see Friebel and Raith (2010), Dessein et al. (2010) and Rantakari (2010) among others for applications to organizational economics.

${ }^{9}$ Persico (2004) considers a similar problem where costly information acquisition is followed by voting. The optimal voting mechanism with costly information acquisition is also considered by Gerardi and Yariv (2007) and Gershkov and Szentes (2009).

${ }^{10}$ In a different setup, Gromb and Martimort (2007) also consider the case where the agent needs to be 


\section{Model}

\section{1 setup}

Consider an organization in which a risk-neutral principal (e.g., a firm owner) hires two riskneutral agents (e.g., workers), each denoted by $i \in\{1,2\}$. Each agent independently chooses a task $x_{i} \in\{L, R\}$ to implement, which stochastically affects the output $y_{i} \in\{0,1\}$. Each agent's task choice is his private information, which cannot be observed by either the other agent or the principal and is hence not contractible. This means that there is no way to force an agent to take an action which is not in his best interest (no authority over the task choice).

The output is either high $\left(y_{i}=1\right)$ or low $\left(y_{i}=0\right)$, depending on the state of nature represented by $(s, a) \in\{L, R\} \times\{G, B\}$. The state of nature is two-dimensional, where $s$ indicates the relative productivity of each task while $a$ indicates the aggregate productivity which captures a common productivity (or demand) shock. When $a=G$, the (aggregate) state is "good" and the output is always high regardless of the task choice. When $a=B$, the state is "bad" and the output is high if and only if the right task, i.e., $x_{i}=s$, is implemented. Letting $p\left(x_{i}, s, a\right):=\operatorname{prob}\left\{y_{i}=1 \mid x_{i}, s, a\right\}$, we thus have

$$
\left\{\begin{array}{l}
p(L, s, G)=p(R, s, G)=1 \text { for } s=L, R \\
p(L, L, B)=p(R, R, B)=1 \text { and } p(L, R, B)=p(R, L, B)=0 .
\end{array}\right.
$$

\subsection{Information acquisition}

The state of nature is not directly observable. The prior distribution of the state is given by

$$
\left\{\begin{array}{l}
\operatorname{prob}\{s=L\}=\operatorname{prob}\{s=R\}=0.5, \\
\operatorname{prob}\{a=G\}=\lambda \text { and } \operatorname{prob}\{a=B\}=1-\lambda,
\end{array}\right.
$$

where $s$ and $a$ are independent of each other. $\lambda$ is one of the key parameters of the model, which measures the salience of the common stochastic shock. We mostly focus on a case where $\lambda$ is strictly positive but relatively small, so that information acquisition constitutes a critical aspect of the production process.

While the state of nature is not freely observable, each agent may observe the relative

motivated to acquire information and reveal it truthfully via contingent contracts. The main difference is that, while we consider communication between the agents, they consider communication between each agent and the principal and in particular examine the effect of potential collusion on the optimal form of organization. 
productivity $s$ by incurring some private cost. ${ }^{11}$ Let $\hat{s}_{i} \in\{L, R, \varnothing\}$ denote the private signal of $s$ observed by agent $i$. Each agent may fail to observe any relevant information with some probability, and this event is denoted by $\hat{s}_{i}=\varnothing$. If $\hat{s}_{i} \neq \varnothing$, then the signal accurately reflects the true state, i.e., $\hat{s}_{i}=s$. For expositional purposes, we say that an agent is "informed" when $\hat{s}_{i} \neq \varnothing$ and "uninformed" when $\hat{s}_{i}=\varnothing$.

The probability that an agent becomes informed depends on how hard he works on acquiring the information. Define $e_{i} \in\{0,1\}$ as the effort level chosen by agent $i$, where $e_{i}=1$ means that the agent chooses to collect evidence of his own. The probability of becoming informed is then given by

$$
\operatorname{prob}\left\{\hat{s}_{i} \neq \varnothing \mid e_{i}\right\}=\operatorname{prob}\left\{\hat{s}_{i}=s \mid e_{i}\right\}=r e_{i}, r \in(0,1)
$$

It should be noted that what we mean by information acquisition in this context is rather broad, including not just the cost of literally gathering information (expertise) but also that of processing and interpreting available evidence or generating ideas, insights and visions that are indispensable when determining the course of action.

The cost of effort is given by $c>0$. We assume that effort is sufficiently valuable for the principal to exclude a trivial equilibrium where the agents are not induced to exert effort. More precisely, we make the following assumption.

\section{Assumption 1}

$$
r \geq \frac{2(1+r) c}{r(1-\lambda)}
$$

As we will see later, this condition is sufficient to rule out the trivial equilibrium.

\subsection{Contracts}

The principal designs and offers a contract to each agent to maximize her expected profit. We assume that each agent's output is the only contractible variable in this environment. ${ }^{12}$ A feasible contract can thus be written as $W_{i}:=\left(w_{i}^{11}, w_{i}^{10}, w_{i}^{01}, w_{i}^{00}\right)$ where $w_{i}^{y_{i} y_{j}}, i \neq j$, denotes the wage to be received by agent $i$ contingent on the outputs $y_{i}$ and $y_{j}$. We impose

\footnotetext{
${ }^{11}$ Note that each agent has no incentive to acquire information about the aggregate state $a$, as long as he observes a signal of the relative productivity $s$, because the knowledge of the aggregate state does not improve his task choice.

${ }^{12}$ To be more precise, we follow the recent literature, e.g., Aghion and Tirole (1996) and Dessein (2001), by assuming that tasks (or states of nature) cannot be described and contracted upon ex ante, so that the principal cannot use a standard mechanism to solicit the private information.
} 
a limited liability condition $w_{i}^{y_{i} y_{j}} \geq 0$, so that any wage payment must be nonnegative. The contracts offered are publicly observable, so that each can observe the other agent's contract.

The nature of a contract is determined largely by how each agent's compensation depends on the other agent's output. For expositional purposes, we use the following terminologies:

- Independent performance evaluation (IPE): $\left(w_{i}^{11}, w_{i}^{01}\right)=\left(w_{i}^{10}, w_{i}^{00}\right)$;

- Relative performance evaluation (RPE): $\left(w_{i}^{11}, w_{i}^{01}\right) \leq\left(w_{i}^{10}, w_{i}^{00}\right)$;

- Joint performance evaluation (JPE): $\left(w_{i}^{11}, w_{i}^{01}\right) \geq\left(w_{i}^{10}, w_{i}^{00}\right)$.

\subsection{Communication and the task choice}

Upon observing a signal, each agent communicates with each other to share the acquired information, if any, and chooses the task to implement. More precisely, each agent simultaneously sends a message $m_{i} \in\{L, R, \varnothing\}$ to the other. The message is costless and unverifiable, so this message game belongs to the class of cheap talk. Given the message and his own observed signal, each agent chooses his task $x_{i} \in\{L, R\}$.

\subsection{Preferences and the timing}

Both the principal and the agents are risk-neutral, where the principal maximizes the expected profit (the expected output minus the expected wage costs) while each agent maximizes the expected wage minus the effort cost. The timing of the model is summarized as follows:

1. The state of nature $(a, s) \in\{G, B\} \times\{L, R\}$ is randomly drawn.

2. The principal offers a contract $W_{i}$ to each agent.

3. Each agent determines the effort level $e_{i} \in\{0,1\}$.

4. Each agent observes a signal $\hat{s}_{i} \in\{L, R, \varnothing\}$.

5. Upon observing $\hat{s}_{i}$, each agent sends a message $m_{i} \in\{L, R, \varnothing\}$ to the other.

6. Upon observing $\hat{s}_{i}$ and $m_{j}, j \neq i$, each agent chooses the task $x_{i} \in\{L, R\}$.

7. The outputs $\left(y_{1}, y_{2}\right) \in\{0,1\}^{2}$ are realized. 


\section{The communication stage}

\subsection{Optimal task choice}

In the communication stage, each agent sends a message $m_{i}$ conditional on the observed signal. Let $M_{i}\left(\hat{s}_{i}\right)$ denote agent $i$ 's message strategy. For the cases we consider, we focus on fully revealing (truth-telling) strategies where $M_{i}\left(\hat{s}_{i}\right)=\hat{s}_{i} \cdot{ }^{13}$ To obtain conditions for truth telling, however, we must first characterize the optimal task choice contingent on the message and the observed signal, under the premise that each agent reports truthfully.

First, if $\hat{s}_{i} \neq \varnothing$, the state is known to be $s=\hat{s}_{i}$. Letting $\sigma_{j}:=\operatorname{prob}\left\{y_{j}=1 \mid \hat{s}_{i}, m_{j}\right\}$, the agent chooses $x_{i}=\hat{s}_{i}$, regardless of the message, if

$$
\sigma_{j} w_{i}^{11}+\left(1-\sigma_{j}\right) w_{i}^{10} \geq \sigma_{j} w_{i}^{01}+\left(1-\sigma_{j}\right) w_{i}^{00}
$$

This condition holds for any $\sigma_{j}$ if $w_{i}^{11} \geq w_{i}^{01}$ and $w_{i}^{10} \geq w_{i}^{00}$. Since this is generally satisfied by any optimal contract as we will see shortly, communication virtually plays no role when the agent is informed.

Communication may matter, on the other hand, when the agent is uninformed. Suppose first that $m_{j} \neq \varnothing$. In this case, the optimal task choice is to follow the other agent's recommendation if

$$
w_{i}^{11} \geq w_{i}^{01}
$$

which is again satisfied by any optimal contract. If $m_{j}=\varnothing$, neither agent has any clue and hence no preference over either task. In this case, we allow the agents to coordinate any way they can to achieve ex ante Pareto-efficient outcomes, if any, when communication is technically feasible. We say that an agent has the incentive to coordinate (on the same task) if

$$
w_{i}^{11}+w_{i}^{00} \geq w_{i}^{10}+w_{i}^{01}
$$

whereas an agent has the incentive to differentiate if this is not satisfied. If (1) is satisfied for both of the agents, it is Pareto-efficient for them to coordinate their task choices $\left(x_{1}=x_{2}\right)$. If it is satisfied for neither, it is then Pareto-efficient to differentiate $\left(x_{1} \neq x_{2}\right)$. Finally, if

\footnotetext{
${ }^{13}$ There are some exceptional cases where it is not possible to achieve full separation. We do not consider these cases because they are not optimal under the maintained assumptions. See Appendix B for more detail.
} 
it is satisfied only for one of the agents, there is no Pareto-efficient outcome, and the agents must randomize and choose each task with equal probability. ${ }^{14}$

\subsection{Strategic information disclosure}

We now turn to the communication stage and explore conditions for truth telling. Since an informed agent cannot be influenced by any message, we can focus on the case where the other agent is uninformed and simply follows whatever is recommended in any truth-telling equilibrium. We check under which an agent has an incentive to reveal truthfully on the basis that $x_{j}=m_{i}$. Given that $\hat{s}_{j}=m_{j}=\varnothing$, there are two cases we need to consider.

Case $1\left(\hat{s}_{i}=\varnothing\right)$ : The agent has no information and hence has no preference over the task choice. If there exists a Pareto-efficient outcome (both agents have an incentive to coordinate or differentiate), it is weakly optimal to report truthfully since the agent is totally indifferent between any messages. This means that under any symmetric contract, an uninformed agent has no incentive to lie. ${ }^{15}$

If one agent has the incentive to coordinate while the other has the incentive to differentiate, on the other hand, truth telling cannot be induced on the equilibrium path. To see this, if an agent reports truthfully, the agents know that they have no relevant information. In this case, they can never agree on who chooses which task and are hence forced to randomize over the tasks. If the agent misrepresents and claims either $m_{i}=L$ or $m_{i}=R$, however, he can induce the other agent to choose $x_{j}=m_{i}$, which allows the agent to coordinate or differentiate. No information can therefore be conveyed in this case.

Case $2\left(\hat{s}_{i} \neq \varnothing\right)$ : This is the case where communication becomes strategic. If the agent reports truthfully, then the other agent yields the high output for sure using that information. This may or may not be in his best interest, depending on the type of contract he faces. To see this, suppose that $\hat{s}_{i}=L$ without loss of generality. The agent has an incentive to lie only when he would like the other agent to choose $x_{j}=R$; the best way to achieve this is to claim $m_{i}=R$. Given this, the agent truthfully discloses his observation iff

$$
w_{i}^{11} \geq w_{i}^{10}
$$

\footnotetext{
${ }^{14}$ This last case would not arise, however, under the three cases we consider.

${ }^{15} \mathrm{~A}$ symmetric contract refers to a case where the same contract is offered to both agents. In this case, the preferences of the two agents are aligned and there must be a Pareto-efficient outcome.
} 
which we refer to as the condition for truth telling. If (2) does not hold, no message can be taken seriously by the other agent, and no information can hence be conveyed.

\section{Optimal organization}

\subsection{Equilibrium with no communication: a benchmark}

We start the analysis with a benchmark case where any form of communication is not feasible between the agents, possibly for some technological reasons. Each agent thus chooses the task independently without ever communicating with the other, as if there exists only one agent. This also precludes the possibility of task coordination when both of the agents are uninformed. For expositional purposes, we refer to this scheme as Independent Production. This benchmark case is instrumental in illuminating the role of communication in the current setup.

Under this scheme, an agent has no choice but to choose the task randomly when he is uninformed. The expected payoff as a function of the effort choices under this scheme, denoted by $\pi_{i}^{\mathrm{I}}$, is then obtained as

$$
\begin{aligned}
\pi_{i}^{I}\left(e_{i}, e_{j}\right)= & r^{2} e_{i} e_{j} w_{i}^{11}+r e_{i}\left(1-r e_{j}\right)\left(\lambda w_{i}^{11}+\frac{(1-\lambda)\left(w_{i}^{11}+w_{i}^{10}\right)}{2}\right) \\
& +r e_{j}\left(1-r e_{i}\right)\left(\lambda w_{i}^{11}+\frac{(1-\lambda)\left(w_{i}^{11}+w_{i}^{01}\right)}{2}\right) \\
& +\left(1-r e_{i}\right)\left(1-r e_{j}\right)\left(\lambda w_{i}^{11}+\frac{(1-\lambda)\left(w_{i}^{11}+w_{i}^{10}+w_{i}^{01}+w_{i}^{00}\right)}{4}\right)-c e_{i} .
\end{aligned}
$$

Taking the other agent's effort choice $e_{j}$ as given, each agent chooses to exert effort iff $\pi_{i}^{\mathrm{I}}\left(1, e_{j}\right) \geq \pi_{i}^{\mathrm{I}}\left(0, e_{j}\right)$, which can be written as

$$
r e_{j} \frac{w_{i}^{11}-w_{i}^{01}}{2}+\left(1-r e_{j}\right) \frac{w_{i}^{11}+w_{i}^{10}-w_{i}^{01}-w_{i}^{00}}{4} \geq \frac{c}{r(1-\lambda)} .
$$

Lemma 1 The optimal contract in the absence of communication is given by

$$
w_{i}^{10}=\frac{4 c}{r(1-r)(1-\lambda)}, w_{i}^{11}=w_{i}^{01}=w_{i}^{00}=0 .
$$

Proof: See Appendix A.

As is well known, in the presence of common stochastic shocks, the optimal contract generally takes the form of RPE, where an agent is compensated based on the difference in 
the outputs, because it filters out common stochastic shocks. ${ }^{16}$ The expected profit under this optimal contract is given by

$$
\begin{aligned}
\Pi^{\mathrm{I}} & =2 \lambda+(1-\lambda)(1+r)-\frac{(1-\lambda)(1-r)(1+r)}{4}\left(w_{1}^{10}+w_{2}^{10}\right) \\
& =2 \lambda+(1-\lambda)(1+r)-\frac{2(1+r) c}{r} .
\end{aligned}
$$

Note that under Assumption 1, this is larger than the expected profit when the agents are not induced to exert effort.

\subsection{Decision making by Committee: bilateral communication}

In the presence of common stochastic shocks, the optimal contract typically takes the form of RPE where the agents are compensated based on the difference in the outputs. One drawback of this type of contract is that by having the agents compete with each other, it necessarily impedes cooperation between them. In this particular context, even when one agent is informed, there is no incentive to reveal truthfully his own observation under this type of contract: the optimal contract in the absence of communication cannot satisfy the condition for truth telling (2). The lack of truthful communication entails an efficiency loss since information is typically non-rival and its value does not depend on the number of people who use it. When the cost of communication is negligibly small (or zero as in this case), it is clearly ex post efficient to share all the relevant information within the organization via communication.

Here, we seek for an equilibrium where both of the agents exert effort to acquire the information and then truthfully report what is observed. We refer to this situation as decision making by Committee, where both agents carry the same weight in the decision-making process ex ante with no restriction on the flow of information (bilateral communication). An apparent virtue of Committee is that it allows the information to flow from an informed party to an uninformed party, making the best use of the existing knowledge within the organization.

In order to facilitate communication between the agents, the condition for truth telling becomes an additional constraint to be satisfied. Once (2) is satisfied, however, the condition for information acquisition also needs to be modified because an uninformed agent may now receive the information via communication. Suppose for now that the agents have the

\footnotetext{
${ }^{16} \mathrm{IPE}, \mathrm{RPE}$ and JPE are all equivalent when $\lambda=0$; for any $\lambda>0$, RPE is strictly better.
} 
incentive to coordinate (which needs to be verified later). The expected payoff, denoted by $\pi_{i}^{\mathrm{C}}$, is then obtained as

$$
\pi_{i}^{\mathrm{C}}\left(e_{i}, e_{j}\right)=\left(r e_{i}+r e_{j}\left(1-r e_{i}\right)\right) w_{i}^{11}+\left(1-r e_{i}\right)\left(1-r e_{j}\right) \frac{(1+\lambda) w_{i}^{11}+(1-\lambda) w_{i}^{00}}{2}-c e_{i} .
$$

The condition for information acquisition is given by

$$
r\left(1-r e_{j}\right)\left(w_{i}^{11}-w_{i}^{00}\right) \geq \frac{2 c}{1-\lambda},
$$

while the condition for truth telling is given by (2). The optimal contract must also satisfy (1) so that the agents indeed have the incentive to coordinate.

Lemma 2 The optimal contract under Committee is given by

$$
w_{i}^{11}=\frac{2 c}{r(1-r)(1-\lambda)}, w_{i}^{10}=w_{i}^{01}=w_{i}^{00}=0 .
$$

Proof: Given that each agent has an incentive to coordinate when uninformed, i.e., (1) is satisfied, the principal's problem under Committee is formulated as

$$
\min _{W_{i}}\left(1-\frac{(1-\lambda)(1-r)^{2}}{2}\right) w_{i}^{11}+\frac{(1-\lambda)(1-r)^{2}}{2} w_{i}^{00},
$$

subject to (2), (4) and the limited liability constraint. It is clear from (4) and the limited liability constraint that $w_{i}^{10}=w_{i}^{01}=w_{i}^{00}=0$. Then, from (4), we obtain $w_{i}^{11}$, which can easily be verified to satisfy (1) and (2).

Q.E.D.

The expected output is maximized under Committee as the output is low only when both fail to acquire the information. This does not necessarily raise the principal's profit, however, since facilitating communication has its own costs. There are three reasons for this. First, to facilitate communication, the agents must be held jointly accountable (JPE), ${ }^{17}$ but JPE is generically a less efficient way to provide incentives in the face of common stochastic shocks. Second, when communication is informative, an uninformed agent can costlessly acquire the information via communication, and there hence arises an incentive to freeride on the other's information. Finally, in the absence of communication, the agents can increase the chance

\footnotetext{
${ }^{17}$ Communicating truthfully in this context can be seen as a form of cooperation. It is now well known that JPE is instrumental in fostering cooperation within an organization. See, e.g., Itoh (1981) and Che and Yoo (2001).
} 
of coordinating their task choices by acquiring the information, which provides an additional incentive. This effect is absent when communication is feasible because the agents can always coordinate their actions via communication even when both of them are uninformed.

This indicates that the ex post efficient rule is not necessarily ex ante efficient as is often emphasized in the literature. The expected profit is

$$
\begin{aligned}
\Pi^{\mathrm{C}} & =2-(1-\lambda)(1-r)^{2}-\frac{2-(1-\lambda)(1-r)^{2}}{2}\left(w_{1}^{11}+w_{2}^{11}\right) \\
& =2-(1-\lambda)(1-r)^{2}-\frac{2\left(2-(1-\lambda)(1-r)^{2}\right) c}{r(1-r)(1-\lambda)}
\end{aligned}
$$

As we will see below, Committee tends to be less profitable than Hierarchy when $\lambda$ or $r$ is close to one, because the cost of adopting JPE is more salient when $\lambda$ is relatively large, and the freeriding incentive intensifies when $r$ is large.

\subsection{Decision making by Hierarchy: unilateral communication}

As we have seen, it is apparently ex post efficient to facilitate communication between the agents when communication is costless as in the current setup. The problem is that it is not ex ante efficient because communication raises the agency cost of inducing costly effort. In the interest of finding a better way to exploit the benefit of communication, we now let the conditions for information acquisition and truth telling hold only for one agent, say agent 1 , so that information only flows from agent 1 to 2 but not the other way around. The chain of command is now hierarchical in that one orders the other what to do, and the other always has the incentive to follow the order.

Since agent 2 exerts no effort, we only need to look at agent 1's incentives. Suppose for now that both of the agents have the incentive to coordinate when uninformed. The expected payoff, denoted by $\pi_{i}^{\mathrm{H}}$, is then given by

$$
\begin{aligned}
\pi_{i}^{H}\left(e_{i}, e_{j}\right)= & r e_{i} w_{i}^{11}+r e_{j}\left(1-r e_{i}\right) \frac{(1+\lambda) w_{i}^{11}+(1-\lambda) w_{i}^{01}}{2} \\
& +\left(1-r e_{i}\right)\left(1-r e_{j}\right) \frac{(1+\lambda) w_{i}^{11}+(1-\lambda) w_{i}^{00}}{2}-c e_{i} .
\end{aligned}
$$

The agent exerts effort iff

$$
w_{i}^{11}-r e_{j} \frac{w_{i}^{11}+w_{i}^{01}}{2}-\left(1-r e_{j}\right) \frac{w_{i}^{11}+w_{i}^{00}}{2} \geq \frac{2 c}{2 r(1-\lambda)} .
$$

We can then show the following. 
Lemma 3 The optimal contract under Hierarchy is

$$
w_{1}^{11}=\frac{2 c}{r(1-\lambda)}, w_{1}^{10}=w_{1}^{01}=w_{1}^{00}=0 \text { and } w_{2}^{11}=w_{2}^{10}=w_{2}^{01}=w_{2}^{00}=0,
$$

which implements $e_{1}=1$ and $e_{2}=0$.

Proof of Lemma 3: We again suppose that both of the agents have an incentive to coordinate, i.e., (1) is satisfied. Since $e_{2}=0$, it is evident that the optimal contract for agent 2 is a trivial one where $w_{2}^{11}=w_{2}^{10}=w_{2}^{01}=w_{2}^{00}=0$. For agent 1 , the principal's problem under Hierarchy is formulated as

$$
\min _{W_{1}}\left(1-\frac{(1-\lambda)(1-r)}{2}\right) w_{1}^{11}+\frac{(1-\lambda)(1-r)}{2} w_{1}^{00}
$$

subject to (2), (5) and the limited liability constraint. It follows from (5) and the limited liability constraint that $w_{i}^{10}=w_{i}^{01}=w_{i}^{00}=0$. Then, from (5), we obtain $w_{1}^{11}$, which can easily be verified to satisfy (1) and (2).

Q.E.D.

The expected profit under Hierarchy is given by

$$
\begin{aligned}
\Pi^{\mathrm{H}} & =1+r+\lambda(1-r)-\left(\lambda+\frac{(1+r)(1-\lambda)}{2}\right) w_{1}^{11} \\
& =1+r+\lambda(1-r)-\frac{(1+\lambda+r(1-\lambda)) c}{r(1-\lambda)} .
\end{aligned}
$$

Note that under Hierarchy, the allocation of responsibility is asymmetric in that agent 1 is given high-powered incentives and also held accountable for agent 2's output (team incentives) while agent 2 is given low-powered incentives and in principle held accountable only for his own output (individual incentives). ${ }^{18}$ There are two virtues of Hierarchy in this setup. First, under Hierarchy, only one agent needs to be motivated to exert effort. Although this arrangement raises the probability that neither agent is informed compared to Committee, it can still capture some benefit of costless communication. Second, it also eliminates the freeriding incentive entirely, which occupies a substantial part of the cost of communication under Committee. By allocating responsibility asymmetrically, agent 1 cannot rely on agent 2, which in turn raises agent 1's motivation to exert effort.

\footnotetext{
${ }^{18}$ In the current specification, agent 2 is actually given no incentives and held accountable for nothing, so that the optimal contract is a special case of IPE. With a simple twist to the basic setup, however, the optimal contract can be made IPE in the strict sense. Suppose that the production process requires costly individual effort $d_{i} \in\{0,1\}$, and the output is given by $y_{i}=p\left(x_{i}, s, a\right) d_{i}$ so that the output is high iff the agent chooses to exert effort for production. In this case, the optimal contract for agent 2 is IPE where the cost of effort is compensated iff his own output is high.
} 


\subsection{Comparison}

We have thus far examined three distinct schemes: (i) Independent Production with no communication, (ii) Committee and (iii) Hierarchy. ${ }^{19}$ We now compare these schemes and see which organization form yields the highest expected profit.

When the outputs are positively correlated, RPE functions well by filtering out common stochastic shocks. It is thus clear that Independent Production, which employs RPE, tends to emerge as the only profitable organizational form when $\lambda$ is relatively large (while satisfying Assumption 1). As $\lambda$ decreases toward zero, on the other hand, the benefit of communication outweighs the cost, and Hierarchy outperforms Independent Production.

Proposition 1 There exists a threshold

$$
\bar{\lambda}^{\mathrm{HI}}=\frac{1+r}{3+r},
$$

such that Hierarchy outperforms Independent Production for $\bar{\lambda}^{\mathrm{HI}} \geq \lambda$.

Proof: It follows from above that

$$
\Pi^{\mathrm{H}}-\Pi^{\mathrm{I}}=\frac{1+r-(3+r) \lambda}{r(1-\lambda)} c .
$$

Solving $\Pi^{\mathrm{H}}-\Pi^{\mathrm{I}} \geq 0$ for $\lambda$ yields the result.

Q.E.D.

It becomes optimal to utilize communication in some way when $\lambda$ is sufficiently small. There are two possibilities as we have seen. One possibility is to have the agents communicate only unilaterally in the fixed direction, as in the case of Hierarchy. When both agents exert effort, however, unilateral communication is not ex post efficient because it is ex ante not clear who ends up with the useful information. The expected output is in general maximized by letting both agents exert effort and communicate bilaterally. When this benefit outweighs the higher agency cost, Committee becomes superior to Hierarchy.

\section{Proposition 2 If}

$$
r^{2}(1-r)^{2}>\left(2(1+r)-(1-r)^{2}\right) c
$$

\footnotetext{
${ }^{19}$ These three schemes do not exhaust all the possible contract forms; however, other forms are rarely optimal under plausible assumptions. See Appendix B for this point.
} 
there exists a threshold

$$
\bar{\lambda}^{\mathrm{CH}}=1+\frac{(1-r) c-\sqrt{(1-r)^{2} c^{2}+8 r^{2}(1+r) c}}{2 r^{2}(1-r)},
$$

such that Committee outperforms Hierarchy for $\bar{\lambda}^{\mathrm{CH}} \geq \lambda$. Otherwise, Hierarchy always outperforms Committee.

Proof: It follows from above that

$$
\Pi^{\mathrm{H}}-\Pi^{\mathrm{C}}=\frac{-r^{2}(1-r)^{2}(1-\lambda)^{2}-(1-r)^{2} c(1-\lambda)+2(1+r) c}{r(1-r)(1-\lambda)} .
$$

Note that this is strictly increasing in $\lambda$, so that $\Pi^{\mathrm{H}}-\Pi^{\mathrm{C}} \geq 0$ is least likely to hold at $\lambda=0$. If

$$
r^{2}(1-r)^{2}>\left(2(1+r)-(1-r)^{2}\right) c
$$

there exists a threshold below which Committee outperforms Hierarchy; otherwise, Hierarchy outperforms Committee for any $\lambda$. Hierarchy outperforms Committee if

$$
-r^{2}(1-r)^{2}(1-\lambda)^{2}-(1-r)^{2} c(1-\lambda)+2(1+r) c \geq 0
$$

which can be written as

$$
\frac{-(1-r) c+\sqrt{(1-r)^{2} c^{2}+8 r^{2}(1+r) c}}{2 r^{2}(1-r)} \geq 1-\lambda .
$$

Q.E.D.

The proposition shows that for any given $c$, Hierarchy tends to dominate Committee when $r$ is close either to one or to the lowerbound implied by Assumption 1. There are two reasons for this. First, since the marginal increase in the output (from Hierarchy to Committee) is $r(1-r)(1-\lambda)$, the benefit diminishes as $r$ approaches either end. Second, as $r$ approaches one, the freeriding incentive intensifies and sharply increases the agency cost. Combined with Proposition 1, the result leads to a sufficient condition for Hierarchy to be the optimal organizational form.

Corollary 1 Hierarchy is optimal if $\bar{\lambda}^{\mathrm{HI}} \geq \lambda$ and $r$ is sufficiently close to zero or one.

We now explore conditions under which Committee becomes optimal. While Hierarchy surely dominates Independent Production for a sufficiently small $\lambda$, the same statement cannot be made for Committee. 


\section{Proposition 3 If}

$$
r(1-r)^{2}>4 c
$$

there exists a threshold

$$
\bar{\lambda}^{\mathrm{CI}}=1+\frac{2 c-2 \sqrt{c^{2}+r^{2} c}}{r^{2}(1-r)}
$$

such that Committee outperforms Independent Production for $\bar{\lambda}^{\mathrm{CI}} \geq \lambda$. Otherwise, Independent Production always outperforms Committee.

Proof: It follows from above that

$$
\Pi^{\mathrm{C}}-\Pi^{\mathrm{I}}=\frac{4((1-\lambda)(1-r)-1)}{r(1-r)(1-\lambda)} c+r(1-r)(1-\lambda) .
$$

Note that this is strictly decreasing in $\lambda$, so that $\Pi^{\mathrm{C}}-\Pi^{\mathrm{I}}<0$ is least likely to hold at $\lambda=0$. If

$$
r(1-r)^{2}>4 c
$$

there exists a threshold below which Committee outperforms Independent Production; otherwise, Independent Production outperforms Committee for any $\lambda$. Committee outperforms Independent Production if

$$
r^{2}(1-r)^{2}(1-\lambda)^{2}+4(1-\lambda)(1-r) c-4 c \geq 0
$$

which can be written as

$$
1-\lambda \geq \frac{-2 c+2 \sqrt{c^{2}+r^{2} c}}{r^{2}(1-r)}
$$

Q.E.D.

From propositions 2 and 3, we can now obtain a sufficient condition for Committee to be optimal. Note that $\lim _{c \rightarrow 0} \bar{\lambda}^{\mathrm{CH}}=1$ and $\lim _{c \rightarrow 0} \bar{\lambda}^{\mathrm{CI}}=1$, which lead to the following statement.

Corollary 2 Committee is optimal if c is sufficiently small. 
In general, Committee works better when the cost of information acquisition is relatively small, which is intuitive because the cost of bilateral communication comes from the increase in the agency cost. This means that if an organization does not need to motivate its members to acquire information, Committee is often an efficient way to make decisions. After all, this is what we expect of a typical committee: in many cases, a committee consists of well informed experts to begin with, and information acquisition is rarely an issue. In such a situation, it is often less optimal to assign more weight on anyone's opinion by restricting the information flow; it thus makes more sense to treat its members more evenly. ${ }^{20}$

\subsection{The first-best allocation: technology or incentives?}

The current analysis shows that Hierarchy often emerges as the optimal contractual arrangement when information acquisition is sufficiently important. Aside from the incentive issues we have discussed, however, there are also other technological factors which influence the optimal number of information sources. One key factor is obviously $r$ : when $r$ is close to one, there is no need to diversify information sources since letting one agent acquire information is almost enough. Similarly, an increase in $\lambda$ diminishes the value of information and hence tends to favor Hierarchy. As it stands, therefore, it is not necessarily clear how important the incentive issues are in deriving our main results.

To single out the effects of those technological factors, we here examine the first-best allocation when there is no information asymmetry, either in information acquisition and communication. In the absence of information asymmetry, the principal can effectively order an agent to exert effort at a cost $c$. Moreover, any information that is obtained in the process will be shared immediately without any strategic concerns. Let $\Pi_{k}, k=0,1,2$, denote the expected profit, which also equals the total surplus, when $k$ agents are induced to exert effort. Straightforward computation then establishes

$$
\begin{gathered}
\Pi_{2}=2-(1-\lambda)(1-r)^{2}-2 c, \\
\Pi_{1}=2-(1-\lambda)(1-r)-c,
\end{gathered}
$$

\footnotetext{
${ }^{20}$ Obviously, the current setup does not apply directly to typical committees where the task choice and the outcome are normally shared among the members. While the model needs some modifications to precisely accommodate this aspect, it does not change the substance of our argument. All that matters in this context is the willingness of each member to share his or hew own information: in the case of typical committees, the members are effectively tied though an exogenously imposed team contract, which gives rise to the three aforementioned costs in the information-acquisition stage.
} 


$$
\Pi_{0}=1+\lambda
$$

It is optimal to induce both of the agents to collect information (Committee) if

$$
(1-\lambda) r(1-r) \geq c
$$

while it is optimal to induce only one agent to do so (Hierarchy) if

$$
(1-\lambda) r>c>(1-\lambda) r(1-r)
$$

As we will see below, (6) is not particularly a stringent condition to be satisfied and holds as long as $c$ is reasonably small.

To conclude this section, we summarize our findings by two figures, each of which depicts which organizational form becomes optimal in the $(r, \lambda)$ space. In each case, the dotted line represents (6) inside of which Committee is the first-best allocation. ${ }^{21}$ It is clear from the figures that Committee is the optimal choice in the absence of information asymmetry except for some extreme cases, suggesting that the incentive issues are the critical force behind our main results. In the presence of information asymmetry, as we have seen, Independent Production is optimal when $\lambda$ is relatively large for the obvious reason. Our interest thus lies in the case where $\lambda$ is not too high. Figure 1 illustrates a situation where the cost of information acquisition is relatively high and the agency problem is hence severer. In this case, Hierarchy is the optimal form of organization for a wide range of parameters. As the cost of information acquisition declines, the range in which Committee becomes optimal enlarges, as depicted in Figure 2.

[Figures 1 and 2]

\section{Conclusion}

This paper considers an environment where the principal can only allocate responsibility via incentive contracts and asks why hierarchies prevail in organizations. The focus of attention is placed on the tension between costly information acquisition and costless communication. In this setting, we show that the optimal incentive scheme is often asymmetric, where one

\footnotetext{
${ }^{21}$ In the figures, we do not indicate the region where no agent is induced to exert effort (Assumption 1 under information asymmetry and (7) under no information asymmetry) for expositional clarity, because the region is negligibly small in either case when $c$ is sufficiently small. These conditions for the trivial equilibrium hold only when $r$ is very close to zero.
} 
agent is given high-powered team incentives while the other is given low-powered individual incentives. Under Hierarchy, the flow of information is restricted to be unilateral and this gives rise to the chain of command pertaining to hierarchial organizations. This asymmetric allocation of responsibility is the optimal way to acquire information and knowledge as a group when the agency cost is sufficiently severe.

\section{References}

Aghion, Philippe and Tirole, Jean, 1997, Formal and Real Authority in Organizations, Journal of Political Economy, 105, 1-29.

Baliga, Sandeep and Sjostrom, Tomas, 1998, Decentralization and Collusion, Journal of Economic Theory, 83, 196-232.

Bolton, Patrick and Dewatripont, Mathias, 1994, The Firm as a Communication Network, Quarterly Journal of Economics, 109, 809-39.

Bolton, Patrick and Dewatripont, Mathias, 2013, Authority in Organizations: A Survey, in Gibbons, Robert and Roberts, John, eds., Handbook of Oraganizational Economics, $342-72$.

Caillaud, Bernard and Tirole, Jean, 2007, Consensus Building: How to Persuade a Group, American Economic Review, 97, 1877-900.

Che, Yeon-Koo and Yoo, Seung-Weon, 2001, Optimal Incentives for Teams, American Economic Review, 91, 525-541.

Choe, Chongwoo and Ishiguro, Shingo, 2012, On the Optimality of Multi-ter Hierarchies: Coordination and Motivation, Journal of Law, Economics, and Organization, 28, 486517.

Crawford, Vincent P. and Sobel, Joel, 1982, Strategic Information Transmission, Econometrica, 50, 1431-51.

Dessein, Wouter, 2002, Authority and Communication in Organizations, Review of Economic Studies, 69, 811-38.

Dessein, Wouter; Garicano, Luis, and Gertner, Robert, 2010, Organizing for Synergies, American Economic Journal: Microeconomics, 4, 77-114. 
Gerardi, Dino and Yariv, Leeat, 2008, Information Acquisition in Committees, Games and Economic Behavior, 62, 436-59.

Gershkov, Alex and Szentes, Balazs, 2009, Optimal Voting Schemes with Costly Information Acquisition, Journal of Economic Theory, 144, 36-68.

Gromb, Denis and Martimort, David, 2007, Collusion and the Organization of Delegated Expertise, Journal of Economic Theory, 137, 271-99.

Grossman, Sanford J. and Hart, Oliver, 1980, Disclosure Laws and Takeover Bids, Journal of Finance, 35, 323-34.

Faure-Grimaud, Antoine; Laffont, Jean-Jacques, and Matimort, David, 2003, Collusion, Delegation and Supervision with Soft Information, Review of Economic Studies, 70, 253-79.

Friebel, Guido and Raith, Michael, 2010, Reosurce Allocation and Organizational Form, American Economic Journal: Microeconomics, 2, 1-33.

Hart, Oliver and Moore, John, 2005, On the Desing of Hierarchies: Coordination versus Specialization, Journal of Political Economy, 113, 675-702.

Itoh, Hideshi, 1991, Incentives to Help in Multi-Agent Situations, Econometrica, 59, 611-636.

Laffont, Jean-Jacques and Martimort, David, 1998, Collusion and Delegation, RAND Journal of Economics, 29, 280-305.

Li, Hao, 2001, A Theory of Conservatism, Journal of Political Economy, 109, 617-36.

Li, Hao and Suen, Wing, 2009, Decision-Making in Committees, Canadian Journal of Economics, 42, 359-92.

Marino, Anthony M.; Matsusaka, John G., and Zabojnik, Jan, 2010, Disobedience and Authority, Journal of Law, Economics, and Organization, 26, 427-59.

Milgrom, Paul R., 1981, Good News and Bad News: Representation Theorems and Applications, Bell Journal of Economics, 12, 380-91.

Mookherjee, Dilip, 2006, Decentralization, Hierarchies and Incentives: A Mechanism Design Perspective, Journal of Economic Literature, 44, 367-90.

Persico, Nicola, 2004, Committee Design with Endogenous Information, Review of Economic Studies, 71, 165-4. 
Radner, Roy, 1993, The Organization of Decentralized Information Processing, Econometrica, $61,1109-46$.

Rajan, Raghuram G. and Zingales, Luigi, 2001, The Firm as a Dedicated Hierarchy: A Theory of the Origins and Growth of Firms, Quarterly Journal of Economics, 116, 805-51.

Rantakari, Heikki, 2010, Organizational Design and Environmental Volatility, mimeo, University of Southern Califormnia.

Sah, Raaj Kumar and Stiglitz, Joseph E., 1988, Committees, Hierarchies and Polyarchies, Economic Journal, 98, 451-70.

Van den Steen, Eric, 2010, Interpersonal Authority in a Theory of the Firm, American Economic Review, 100, 466-90.

Van Zandt, Timothy, 1999, Real-Time Decentralized Information Processing as a Model of Organizations with Boundedly Rational Agents, Review of Economic Studies, 66, 633-58.

\section{Appendix A: Proofs}

Proof of Lemma 1: Provided that it is optimal to implement $e_{i}=1$, the principal's problem in the absence of communication is defined as

$$
\begin{aligned}
\min _{W_{i}} & \frac{4 \lambda+(1-\lambda)\left(4 r+(1-r)^{2}\right)}{4} w_{i}^{11}+\frac{(1-\lambda)\left(2 r(1-r)+(1-r)^{2}\right)}{4}\left(w_{i}^{10}+w_{i}^{01}\right) \\
& +\frac{(1-\lambda)(1-r)^{2}}{4} w_{i}^{00},
\end{aligned}
$$

subject to (3) and the limited liability constraint. It directly follows from (3) that $w_{i}^{01}=$ $w_{i}^{00}=0$ at the optimal solution. This leaves us two possibilities, either $w_{i}^{11}>w_{i}^{10}=0(\mathrm{JPE})$ or $w_{i}^{10}>w_{i}^{11}=0$ (RPE). A candidate contract under JPE, denoted by $\tilde{w}_{i}^{11}$, is

$$
\tilde{w}_{i}^{11}=\frac{4 c}{r(1+r)(1-\lambda)} \text {. }
$$

Similarly, a candidate contract under RPE, denoted by $\tilde{w}_{i}^{10}$, is

$$
\tilde{w}_{i}^{10}=\frac{4 c}{r(1-r)(1-\lambda)} .
$$

RPE yields higher profit than JPE if

$$
\frac{4 \lambda+(1-\lambda)\left(4 r+(1-r)^{2}\right)}{4} \tilde{w}_{i}^{11} \geq \frac{(1-\lambda)\left(2 r(1-r)+(1-r)^{2}\right)}{4} \tilde{w}_{i}^{10} .
$$


This can be written as

$$
\frac{4 \lambda+(1-\lambda)\left(4 r+(1-r)^{2}\right)}{1+r} \geq \frac{(1-\lambda)\left(2 r(1-r)+(1-r)^{2}\right)}{1-r}
$$

which is further simplified to

$$
\lambda(1-r) \geq 0
$$

This shows that RPE is generically better than JPE (the two are equivalent only when $\lambda=0$ ).

Q.E.D.

\section{Appendix B: Other Contractual Arrangements}

In the analysis, we examine only three contractual arrangements, which apparently do not exhaust all the possibilities. Note that there are two incentive compatibility constraints one for information acquisition (hereafter IA) and the other for truth telling (TT). Since the condition for truth telling matters only when the condition for information acquisition is satisfied, there are generically six possible contract forms as summarized below:

1. IA and TT are satisfied for both agents (Committee).

2. IA is satisfied for both agents but TT is satisfied for one agent.

3. IA is satisfied for both agents but TT is satisfied for neither.

4. IA and TT are satisfied for one agent (Hierarchy).

5. IA is satisfied for one agent but TT is satisfied for neither.

6. IA is satisfied for neither.

Cases 1 and 4 are already analyzed. We have also seen that it is optimal to let the agents acquire the information in the absence of communication, which rules out cases 5 and 6 . This implies that we only need to consider cases 2 and 3 .

\section{Case 2}

In this case, both of the agents exert effort to acquire the information, but only one of them reports it truthfully. We refer to this situation as Partial Delegation, since the flow of information is unilateral as in the case of Hierarchy but the subordinate acts on his own information whenever he has one. For the sake of the argument, suppose that TT is satisfied 
for agent 1 but not for agent 2 . To derive the optimal contract within this class, we need to consider four distinct possibilities.

Case 2-1. Only agent 1 has the incentive to coordinate: In this case, fully separating communication cannot be implemented because there is no incentive to report truthfully when $\hat{s}_{1}=\varnothing$. For communication to be meaningful, it is then necessary for agent 2 to follow agent 1 's message when he is uninformed. Given that $\hat{s}_{2}=\varnothing$, this condition can be written as

$$
\begin{aligned}
& r e_{1} w_{2}^{11}+\left(1-r e_{1}\right)\left(\lambda w_{2}^{11}+(1-\lambda) \frac{w_{2}^{11}+w_{2}^{00}}{2}\right) \\
& \geq r e_{1}\left(\lambda w_{2}^{11}+(1-\lambda) w_{2}^{01}\right)+\left(1-r e_{1}\right)\left(\lambda w_{2}^{11}+(1-\lambda) \frac{w_{2}^{10}+w_{2}^{01}}{2}\right),
\end{aligned}
$$

which is simplified to

$$
\left(1+r e_{1}\right)\left(w_{2}^{11}-w_{2}^{01}\right) \geq\left(1-r e_{1}\right)\left(w_{2}^{10}-w_{2}^{00}\right) .
$$

The condition for truth telling for agent 1 is the same as before: the agent reports truthfully iff $w_{1}^{11} \geq w_{1}^{10}$.

Given that these conditions are satisfied, the expected payoff for agent 1 is obtained as

$$
\begin{aligned}
\pi_{1}\left(e_{1}, e_{2}\right)= & r e_{1} w_{1}^{11}+r e_{2}\left(1-r e_{1}\right) \frac{(1+\lambda) w_{1}^{11}+(1-\lambda) w_{1}^{01}}{2} \\
& +\left(1-r e_{1}\right)\left(1-r e_{2}\right) \frac{(1+\lambda) w_{1}^{11}+(1-\lambda) w_{1}^{00}}{2}-c e_{1} .
\end{aligned}
$$

The agent exerts effort iff

$$
w_{1}^{11}-r e_{2} w_{1}^{01}-\left(1-r e_{2}\right) w_{1}^{00} \geq \frac{2 c}{r(1-\lambda)} .
$$

A candidate contract is

$$
w_{1}^{11}=\frac{2 c}{r(1-\lambda)}, w_{1}^{10}=w_{1}^{01}=w_{1}^{00}=0
$$

which also satisfies the condition for truth telling. Under this contract, the agent also has an incentive to coordinate.

Similarly, the expected payoff for agent 2 is obtained as

$$
\begin{aligned}
\pi_{2}\left(e_{2}, e_{1}\right)= & r e_{1} w_{2}^{11}+r e_{2}\left(1-r e_{1}\right) \frac{(1+\lambda) w_{2}^{11}+(1-\lambda) w_{2}^{10}}{2} \\
& +\left(1-r e_{2}\right)\left(1-r e_{1}\right) \frac{(1+\lambda) w_{1}^{11}+(1-\lambda) w_{1}^{00}}{2}-c e_{2} .
\end{aligned}
$$


The agent exerts effort iff

$$
\left(1-r e_{1}\right)\left(w_{2}^{10}-w_{2}^{00}\right) \geq \frac{2 c}{r(1-\lambda)}
$$

We have also assume that the agent has an incentive to differentiate, which means that

$$
w_{2}^{10}-w_{2}^{00}>w_{2}^{11}-w_{2}^{01}
$$

The optimal contract within this class must satisfy (8), (9) and (10). Since $e_{1}=1$, all of these condition together imply

$$
w_{2}^{10}-w_{2}^{00}>w_{2}^{11}-w_{2}^{01} \geq \frac{1-r}{1+r}\left(w_{2}^{10}-w_{2}^{00}\right) \geq \frac{2 c}{r(1+r)(1-\lambda)} .
$$

A candidate contract is thus

$$
w_{2}^{11}=\frac{2 c}{r(1+r)(1-\lambda)}, w_{2}^{10}=\frac{2 c}{r(1-r)(1-\lambda)}, w_{1}^{01}=w_{1}^{00}=0 .
$$

Under this contract, the agent has no incentive to report truthfully, as assumed.

We now show that this contract cannot be optimal. The expected profit under this arrangement, denoted by $\Pi^{\mathrm{P}}$, is

$$
\begin{aligned}
\Pi^{\mathrm{P}}= & 2 r+r(1-r)+\frac{(1-r)(2-r)(1+\lambda)}{2}-\left(r+\frac{(1-r)(1+\lambda)}{2}\right) \frac{2 c}{r(1-r)(1-\lambda)} \\
& -\left(r+\frac{(1-r)(1+\lambda)}{2}\right) \frac{2 c}{r(1+r)(1-\lambda)}-\frac{r(1-r)(1-\lambda)}{2} \frac{2 c}{r(1-r)(1-\lambda)} .
\end{aligned}
$$

Let the expected profit under each arrangement be decomposed into the expected output $Y^{k}$ and the expected wage cost $W^{k}$, such that $\Pi^{k}=Y^{k}-W^{k}, k=H, C, P$. Hierarchy outperforms Partial Delegation if $Y^{\mathrm{H}}-W^{\mathrm{H}} \geq Y^{\mathrm{P}}-W^{\mathrm{P}}$ while Committee outperforms Partial Delegation if $Y^{\mathrm{C}}-W^{\mathrm{C}} \geq Y^{\mathrm{P}}-W^{\mathrm{P}}$. Since

$$
Y^{\mathrm{C}}-Y^{\mathrm{P}}=Y^{\mathrm{P}}-Y^{\mathrm{H}}=\frac{r(1-r)(1-\lambda)}{2},
$$

Partial Delegation cannot be optimal if $2 W^{\mathrm{P}} \geq W^{\mathrm{C}}+W^{\mathrm{H}}$, which can be written as

$$
\frac{4(2 r+(1-r)(1+\lambda))}{r(1-r)(1+r)(1-\lambda)}+2 \geq \frac{4-2(1-\lambda)(1-r)^{2}}{r(1-r)(1-\lambda)}+\frac{1+\lambda+r(1-\lambda)}{r(1-\lambda)} .
$$

With some algebra obtain

$$
1 \geq \frac{(1+r)\left(4-2(1-\lambda)(1-r)^{2}\right)+(1-r)(1+r)(1+\lambda)-8 r-4(1-r)(1+\lambda)}{r(1-r)(1+r)(1-\lambda)} .
$$


which is further simplified to

$$
1 \geq \frac{(1+r)(3 \lambda-2 r(1-\lambda)-1)-4 \lambda}{r(1+r)(1-\lambda)} \Leftrightarrow(1+r)^{2} \geq\left(4 r+r^{2}-1\right) \lambda .
$$

One can then easily verify that this condition holds for any $\lambda \in[0,1]$, which proves that Delegated Hierarchy cannot be optimal in this case.

Case 2-2. Only agent 1 has the incentive to differentiate: Again, in this case, fully separating communication cannot be implemented because there is no incentive to report truthfully when $\hat{s}_{1}=\varnothing$. We thus need the same condition as above, i.e., it is necessary for agent 2 to follow agent 1's message when he is uninformed. Given that $\hat{s}_{2}=\varnothing$, this condition can be written as

$$
\begin{aligned}
& r e_{1} w_{2}^{11}+\left(1-r e_{1}\right)\left(\lambda w_{2}^{11}+(1-\lambda) \frac{w_{2}^{10}+w_{2}^{01}}{2}\right) \\
& \geq r e_{1}\left(\lambda w_{2}^{11}+(1-\lambda) w_{2}^{01}\right)+\left(1-r e_{1}\right)\left(\lambda w_{2}^{11}+(1-\lambda) \frac{w_{2}^{11}+w_{2}^{00}}{2}\right),
\end{aligned}
$$

which is simplified to

$$
\left(1+r e_{1}\right)\left(w_{2}^{11}-w_{2}^{01}\right) \geq\left(1-r e_{1}\right)\left(w_{2}^{00}-w_{2}^{10}\right) .
$$

The condition for truth telling for agent 1 is the same as before: the agent reports truthfully iff $w_{1}^{11} \geq w_{1}^{10}$.

Given that these conditions are satisfied, the expected payoff for agent 1 is obtained as

$$
\begin{aligned}
\pi_{1}\left(e_{1}, e_{2}\right)= & r e_{1} w_{1}^{11}+r e_{2}\left(1-r e_{1}\right) \frac{(1+\lambda) w_{1}^{11}+(1-\lambda) w_{1}^{01}}{2} \\
& +\left(1-r e_{1}\right)\left(1-r e_{2}\right) \frac{2 \lambda w_{1}^{11}+(1-\lambda)\left(w_{1}^{10}+w_{1}^{01}\right)}{2}-c e_{1} .
\end{aligned}
$$

The agent exerts effort iff

$$
r e_{2}\left(w_{1}^{11}-w_{1}^{01}\right)+\left(1-r e_{2}\right)\left(2 w_{1}^{11}-w_{1}^{10}-w_{1}^{01}\right) \geq \frac{2 c}{r(1-\lambda)} .
$$

Since $w_{1}^{10}+w_{1}^{01} \geq w_{1}^{11}+w_{1}^{00}$ (the incentive to differentiate), a necessary condition for this is

$$
r e_{2}\left(w_{1}^{11}-w_{1}^{01}\right)+\left(1-r e_{2}\right)\left(w_{1}^{11}-w_{1}^{00}\right) \geq \frac{2 c}{r(1-\lambda)} .
$$

A candidate contract is

$$
w_{1}^{11}=w_{1}^{10}=\frac{2 c}{r(1-\lambda)}, w_{1}^{01}=w_{1}^{00}=0
$$


which also satisfies the condition for truth telling.

Similarly, the expected payoff for agent 2 is obtained as

$$
\begin{aligned}
\pi_{2}\left(e_{2}, e_{1}\right)= & r e_{1} w_{2}^{11}+r e_{2}\left(1-r e_{1}\right) \frac{(1+\lambda) w_{2}^{11}+(1-\lambda) w_{2}^{10}}{2} \\
& +\left(1-r e_{2}\right)\left(1-r e_{1}\right) \frac{2 \lambda w_{1}^{11}+(1-\lambda)\left(w_{2}^{10}+w_{1}^{01}\right)}{2}-c e_{2} .
\end{aligned}
$$

The agent exerts effort iff

$$
\left(1-r e_{1}\right)\left(w_{2}^{11}-w_{2}^{01}\right) \geq \frac{2 c}{r(1-\lambda)} .
$$

Since the condition for truth telling must not hold for agent 2 , a candidate contract is

$$
w_{2}^{11}=w_{2}^{10}=\frac{2 c}{r(1-r)(1-\lambda)}, w_{1}^{01}=w_{1}^{00}=0 .
$$

These contracts are, however, clearly less profitable than the ones obtained in the previous case, implying that Partial Delegation is not optimal in this case.

Case 2-3. Both agents have the incentive to coordinate: The condition for information acquisition is the same as in case $2-1$. The condition for agent 1 is given by

$$
w_{1}^{11}-r e_{2} w_{1}^{01}-\left(1-r e_{2}\right) w_{1}^{00} \geq \frac{2 c}{r(1-\lambda)} .
$$

while that for agent 2 is

$$
\left(1-r e_{1}\right)\left(w_{2}^{10}-w_{2}^{00}\right) \geq \frac{2 c}{r(1-\lambda)}
$$

Since full revelation is possible in this case, the incentive for agent 2 to follow agent 1's message is not necessary. Agent 2 must have the incentive to coordinate, however, and that requires

$$
w_{2}^{11}+w_{2}^{00} \geq w_{2}^{10}+w_{2}^{01}
$$

Note that this restriction is more stringent than (8), so that the resulting profit must be less than that in case $2-1$.

Case 2-4. Both agents the incentive to differentiate: The condition for information acquisition is the same as in case $2-2$. The condition for agent 1 is given by

$$
r e_{2}\left(w_{1}^{11}-w_{1}^{01}\right)+\left(1-r e_{2}\right)\left(2 w_{1}^{11}-w_{1}^{10}-w_{1}^{01}\right) \geq \frac{2 c}{r(1-\lambda)} .
$$


while that for agent 2 is

$$
\left(1-r e_{1}\right)\left(w_{2}^{11}-w_{2}^{01}\right) \geq \frac{2 c}{r(1-\lambda)} .
$$

Since full revelation is possible in this case, the incentive for agent 2 to follow agent 1's message is not necessary. Agent 1 must have the incentive to differentiate, however, and that requires

$$
w_{1}^{10}+w_{1}^{01} \geq w_{1}^{11}+w_{1}^{00} .
$$

Moreover, the condition for truth telling must not hold for agent 2. These additional requirements are the same as in case 2-2, leading to the same outcome.

\section{Case 3}

This situation is almost identical to Independent Production, except that communication is now feasible, which allows them to coordinate on the task choice while exchanging no information about the state of nature. Suppose first that each agent has the incentive to differentiate. We can then consider the following strategy for each agent.

- When $\hat{s}_{i} \neq \varnothing, m_{i}=L$ or $m_{i}=R$ with equal probability (a babbling message);

- When $\hat{s}_{i}=\varnothing, m_{i}=\varnothing$.

One can verify that neither agent has the incentive to deviate from this strategy. Under this strategy, the expected payoff, denoted by $\tilde{\pi}_{i}^{\mathrm{I}}$, is

$$
\begin{aligned}
\tilde{\pi}_{i}^{I}\left(e_{i}, e_{j}\right)= & r^{2} e_{i} e_{j} w_{i}^{11}+r e_{i}\left(1-r e_{j}\right)\left(\lambda w_{i}^{11}+\frac{(1-\lambda)\left(w_{i}^{11}+w_{i}^{10}\right)}{2}\right) \\
& +r e_{j}\left(1-r e_{i}\right)\left(\lambda w_{i}^{11}+\frac{(1-\lambda)\left(w_{i}^{11}+w_{i}^{01}\right)}{2}\right) \\
& +\left(1-r e_{i}\right)\left(1-r e_{j}\right)\left(\lambda w_{i}^{11}+\frac{(1-\lambda)\left(w_{i}^{10}+w_{i}^{01}\right)}{2}\right)-c e_{i} .
\end{aligned}
$$

The agent chooses to exert effort iff

$$
\frac{w_{i}^{11}-w_{i}^{01}}{2} \geq \frac{c}{r(1-\lambda)} .
$$

Since the agents must the incentive to differentiate, we must also have

$$
w_{i}^{10}+w_{i}^{01} \geq w_{i}^{11}+w_{i}^{00} .
$$


The optimal contract within this class is hence

$$
w_{i}^{11}=w_{i}^{10}=\frac{2 c}{r(1-\lambda)}, w_{i}^{01}=w_{i}^{00}=0
$$

but this is clearly less efficient than Independent Production. To see this, note that the expected output under this contract is exactly the same as that under Independent Production because any task coordination when both agents are uninformed does not increase the expected output. Independent Production then dominates this if

$$
\left(r^{2}+r(1-r)(1+\lambda)+(1-r)^{2} \lambda+\frac{(1-r)(1-\lambda)}{2}\right) \frac{2 c}{r(1-\lambda)} \geq \frac{(1+r) c}{r},
$$

which can be shown to hold.

Now suppose that both of the agents have the incentive to coordinate. The same strategy considered above works, and under this strategy, the expected payoff is

$$
\begin{aligned}
\tilde{\pi}_{i}^{I}\left(e_{i}, e_{j}\right)= & r^{2} e_{i} e_{j} w_{i}^{11}+r e_{i}\left(1-r e_{j}\right)\left(\lambda w_{i}^{11}+\frac{(1-\lambda)\left(w_{i}^{11}+w_{i}^{10}\right)}{2}\right) \\
& +r e_{j}\left(1-r e_{i}\right)\left(\lambda w_{i}^{11}+\frac{(1-\lambda)\left(w_{i}^{11}+w_{i}^{01}\right)}{2}\right) \\
& +\left(1-r e_{i}\right)\left(1-r e_{j}\right)\left(\lambda w_{i}^{11}+\frac{(1-\lambda)\left(w_{i}^{11}+w_{i}^{00}\right)}{2}\right)-c e_{i} .
\end{aligned}
$$

The agent chooses to exert effort iff

$$
r e_{j} \frac{w_{i}^{11}-w_{i}^{01}}{2}+\left(1-r e_{j}\right) \frac{w_{i}^{10}-w_{i}^{00}}{2} \geq \frac{c}{r(1-\lambda)} .
$$

Since the agents must the incentive to coordinate, we must also satisfy (1). The principal's problem is then defined as

$$
\begin{aligned}
\min _{W_{i}} & \frac{2 r^{2}+\left(1-r^{2}\right)(1+\lambda)}{2} w_{i}^{11}+\frac{r(1-r)(1-\lambda)}{2}\left(w_{i}^{10}+w_{i}^{01}\right) \\
& +\frac{(1-\lambda)(1-r)^{2}}{2} w_{i}^{00}
\end{aligned}
$$

subject to the incentive constraints. In the absence of (1), the optimal contract would have $w_{i}^{11}=0$. The optimal contract within this class is thus given by

$$
w_{i}^{11}=w_{i}^{10}=\frac{2 c}{r(1-\lambda)}, w_{i}^{01}=w_{i}^{00}=0
$$

which yields exactly the same profit as the one derived above (for the case where both agents have the incentive to differentiate). 


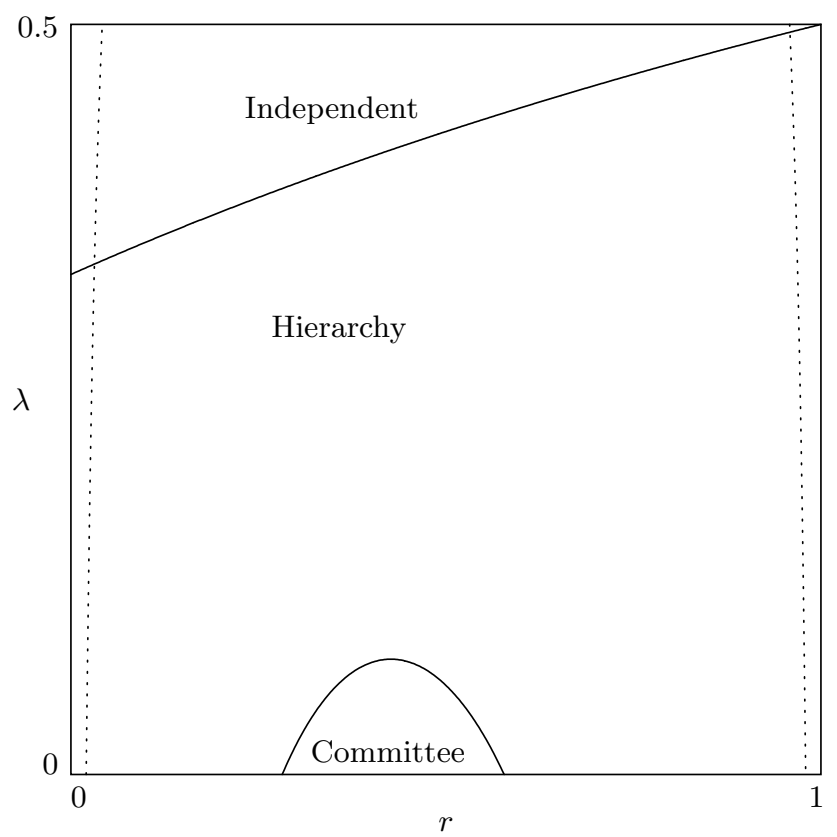

Figure 1: Optimal organization when the cost of information acquisition is high $(c=0.02)$ 


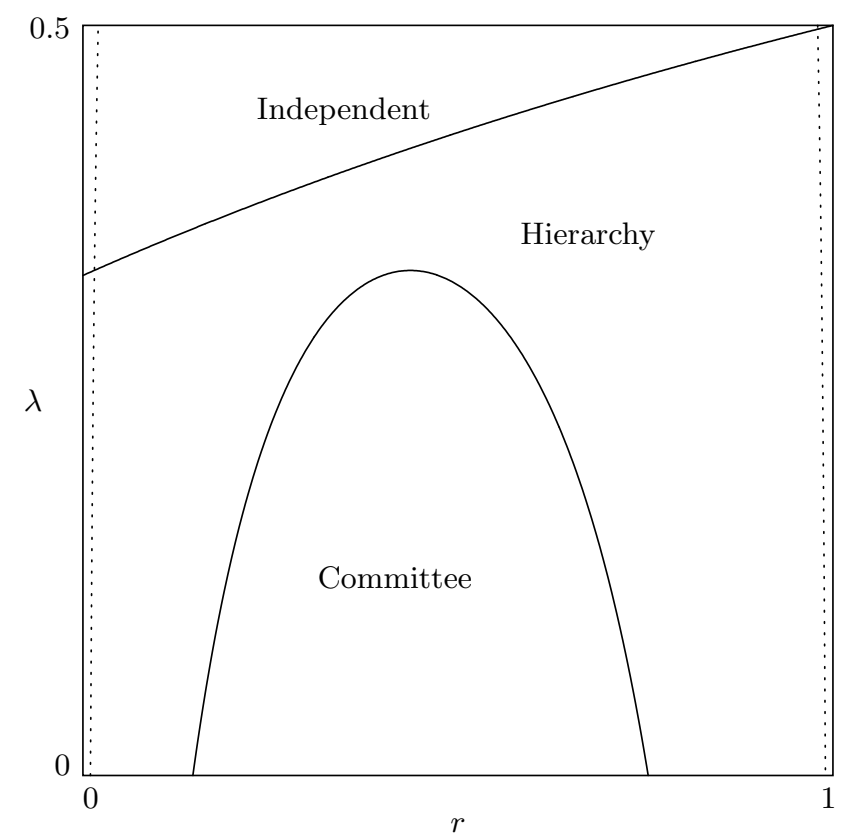

Figure 2: Optimal organization when the cost of information acquisition is low $(c=0.01)$ 